\title{
IMMERSED BOUNDARY METHOD APPLICATION AS A WAY TO BUILD A SIMPLIFIED FLUID-STRUCTURE MODEL
}

\author{
JONATAS EMMANUEL BORGES ${ }^{1}$, MARCOS ANTONIO DE SOUZA LOURENÇO ${ }^{2}$, \\ ELIE LUIS MARTÍNEZ PADILLA ${ }^{3} \&$ CHRISTOPHER MICALLEF ${ }^{4}$ \\ ${ }^{1}$ Federal University of Mato Grosso, Brazil \\ ${ }^{2}$ Federal University of Technology - Paraná, Brazil \\ ${ }^{3}$ Federal University of Uberlândia, Brazil \\ ${ }^{4}$ University of Malta, Malta
}

\begin{abstract}
A simplified fluid-structure interaction model, consisting of a cylinder tethered by a spring system interacting dynamically with an incompressible two-dimensional lid-driven cavity flow, is solved using the immersed boundary method. Results show that when the spring forces are weaker than the fluid drag force, the springs stretch freely and the cylinder motion is the direct result of the fluid dynamics action. For higher values of spring forces, the cylinder motion reaches a maximum displacement, and the spring forces induce the cylinder to an oscillatory movement damped by the fluid drag forces. Subsequently the amplitude of the displacement decreases. The cylinder motion is restricted within the mainstream fluid flow, where the maximum displacement reduces as the Reynolds number increases. Keywords: computational fluid dynamics, immersed boundary method, fluid-structure interaction.
\end{abstract}

\section{INTRODUCTION}

A lot of research is going on in the field of computational fluid dynamics (CFD) in order to balance the need to model increasingly complex boundary conditions and achieve highly accurate results at a minimum amount of computational time and resources [1]. The great majority of engineering fluid flow problems are characterized by complex geometries, which are often associated with the presence of solid, moving or flexible walls.

Most situations encountered in engineering practice involve complex geometries which are not fit for Cartesian grids and the choice of grid is not at all trivial. In order to deal with complex geometries, whole families of numerical methods have been developed. Body-fitted coordinate grids, such as curvilinear grids, non-orthogonal grids and non-structured grids, are usually employed. Unfortunately grid generation for complex geometries is generally an issue since it consumes a lot of user time especially when commercial codes are not employed.

One alternative to body-fitting meshes is the increasingly popular immersed boundary (IB) method. The immersed boundary method was pioneered by Peskin [2] in a paper on simulation of blood flowing through cardiac valves, where biological deformable tissue interact with the viscous flow. Since then, the method has experienced successive refinements and modifications, attracting considerable interest as an important tool for solving general fluid-fluid or fluid-solid interaction problems [3]-[6].

The distinguishing quality of an IB method is to simulate an interface inside a flow by adding a force field to discretised Navier-Stokes equations. The embedded interface is represented by an arbitrary Lagrangian mesh whereas the flow domain is usually discretised by a simple Eulerian orthogonal grid. An interpolation function transfers the information from one domain to another and back.

Borges et al. [7] applied the direct forcing method [4] in order to investigate Newtonian laminar flow through a three-dimensional sudden contraction using Cartesian meshes. To 
compute the transfer functions between the Eulerian and Lagrangian grids, the moving-leastsquare (MLS) approximation [5] was employed. Comparison between numerical and experimental velocity profiles has shown good agreement.

The advantages of IB method are interesting for fluid-structure interaction where the body motion or body deformation does not imply the need for grid restructuring at every single time step. Fluid-structure interaction (FSI) problems occur in many areas of engineering and represent one of the greatest challenges of our time.

Fluid-structure interaction problems can be solved either monolithically, by solving the flow and structure equations simultaneously, or in a partitioned way, having separate solvers for the fluid flow equations and the structural equations. The main advantage of the monolithic approach is that no coupling iterations within the time step are required. On the other hand, existing flow solvers and structural solvers can be employed in a partitioned simulation, reducing software development efforts and improving manageability. Furthermore, this approach can solve FSI problems that do not require matching meshes at the interfaces between the solid and the fluid [8]. Degroote et al. [9], [10] compared the monolithic and partitioned approaches and reported that the performance of the two approaches was problem-dependent.

Nowadays, FSI simulations are widely used in industry. In the aerospace industry, FSI is used in order to study flutter on control surfaces, lift devices and also turbo machinery blades. On the other hand FSI is used in the renewable energy industry to investigate wind turbine behavior and also wave-energy converters. Parachute dynamics is another interesting field as large deformations appear. The design and analysis of artificial heart valves, the prediction of the rupture of aneurysms or of the outcome of surgery also rely extensively on FSI simulations in patient-specific geometries [9].

The difficulty of developing mathematical models which adequately represent the complexity of fluid-structure interaction problems, has led to simplified physical models in order to initiate the study and for a better understanding of the phenomena involved. One of the strategies for the development of simplified models was the proposal where the structure is represented by a body, spring and damper assembly. As an example, important knowledge has been acquired on the vortex-induced vortex vibration problem (VIV) considering the flow on oscillating cylinders [11]-[13].

Campregher [14] employed the physical virtual model originally proposed by Lima e Silva et al. [3] to study the flow around a stationary cylinder. Results were validated against well reported benchmarks in published literature. The study was then extended to a fluidstructure interaction problem using the partitioned approach. The dynamic system chosen was composed by a cylinder tethered by springs, immersed in the flow. This study produced interesting results.

This work presents a simplified fluid-structure problem where the cylinder, represented by the multi-direct forcing of Wang et al. [6], is tethered by springs immersed in an incompressible two-dimensional Newtonian lid-driven cavity flow, where fluid-structure interaction takes places.

\section{MATHEMATICAL DESCRIPTION}

\subsection{Governing equations for fluid flow}

This work is restricted to incompressible Newtonian fluid flows which are governed by Navier-Stokes and continuity equations. In the physical space and relative to an inertial reference frame these equations are 


$$
\begin{gathered}
\frac{\partial u_{i}}{\partial t}+\frac{\partial\left(u_{i} u_{j}\right)}{\partial x_{j}}=-\frac{1}{\rho} \frac{\partial p}{\partial x_{i}}+\frac{\partial}{\partial x_{j}}\left[v\left(\frac{\partial u_{i}}{\partial x_{j}}+\frac{\partial u_{j}}{\partial x_{i}}\right)\right]+\frac{f_{i}}{\rho}, \\
\frac{\partial u_{j}}{\partial x_{j}}=0,
\end{gathered}
$$

where $u_{i}(\vec{x}, t)$ and $p(\vec{x}, t)$ are the velocity and pressure fields, respectively; $f_{i}(\vec{x}, t)$ is the force field, $\rho$ is the density and $v$ is the kinematic viscosity. The term $f_{i}(\vec{x}, t)$ is the external force exerted on the flow field which is the mutual interaction force between fluid and immersed boundary expressed as follows

$$
f_{i}(\vec{x}, t)=\int_{\Gamma} F_{k}\left(\overrightarrow{x_{k}}, t\right) \delta\left(\vec{x}-\overrightarrow{x_{k}}\right) d x_{k},
$$

where $\delta\left(\overrightarrow{x_{k}}-\vec{x}\right)$ is a distribution function, $\overrightarrow{x_{k}}$ is the position of the Lagrangian markers set at the immersed boundary, $\vec{x}$ is the position of the computational Eulerian mesh and $F_{k}\left(\overrightarrow{x_{k}}, t\right)$ is the force exerted on the Lagrangian marker $\overrightarrow{x_{k}}$. Fig. 1 illustrates the distribution of the Lagrangian force to adjacent Eurelian volumes.

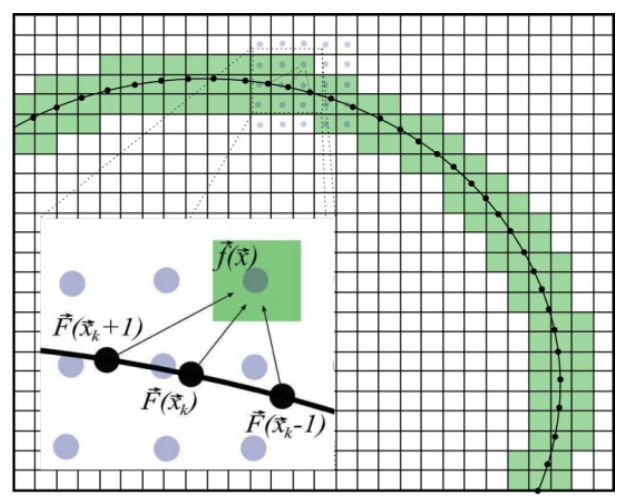

Figure 1: Distribution of the Lagrangian force to adjacent Eulerian volumes (shaded).

\section{NUMERICAL METHODS}

The governing eqns (1) and (2) are discretized by the finite volume method, where the advective and diffusive terms are discretised using a central differencing scheme, in a staggered arrangement, as proposed by Patankar [15]. In a simple rectilinear mesh discretization, the components $\mathrm{u}$ and $\mathrm{v}$ are positioned in the volume's normal faces in the $\mathrm{x}$ and y directions respectively. Scalar values, as the pressure, are located at the volume center. The discretization in time was performed by a Runge-Kutta second order scheme. Pressurevelocity coupling uses the two-step fractional method of Kim and Moin [16]. The solution of the Poisson equation is performed by Successive Over Relaxation (SOR) iterative method.

The velocity field is initially estimated as $u_{i}^{*}$, in eqn (4)

$$
u_{i}^{*}=u_{i}^{t}+\left(-\frac{1}{\rho} \frac{\delta p^{t}}{\delta x_{i}}-\frac{\delta\left(u_{i} u_{j}\right)}{\delta x_{j}}+v \frac{\delta^{2} u_{k i}}{\delta x_{k j} \delta x_{k j}}\right)^{l} \frac{\Delta t}{2-l} \quad l=0,1 .
$$

The velocity $\mathrm{u}_{\mathrm{i}}^{\mathrm{t}+\Delta \mathrm{t}}$ is estimated using

$$
u_{i}^{t+\Delta t}=u_{i}^{*}-\Delta t \frac{\delta p^{\prime}}{\delta x_{i}}
$$


where the pressure fluctuation $p^{\prime}$ is determined by the solution of a Poisson equation in the form of

$$
\frac{\delta}{\delta x_{i}}\left(\frac{\delta p^{\prime}}{\delta x_{i}}\right)=\frac{\rho}{\Delta t} \frac{\delta u_{i}^{*}}{\delta x_{i}}
$$

leading to the pressure $p^{t+\Delta t}$ estimation through

$$
p^{t+\Delta t}=p^{\prime}+p^{t}
$$

3.1 Mathematical model for the immersed interface

The Lagrangian force field is calculated by the direct forcing method proposed by Uhlmann [4]. This method enables the modeling of the wall boundary condition in the immersed interface without introducing any ad hoc constants. The Lagrangian force, $F_{i}\left(\overrightarrow{x_{k}}, t\right)$, is calculated through the momentum equation (eqn (8)) over a fluid particle in the fluid-solid interface. A force $F_{i}\left(\overrightarrow{x_{k}}, t\right)$ is imposed on the Lagrangian marker to equate its velocity to the desired velocity $u_{i}$ IBM at the immersed boundary

$$
\frac{F_{i}\left(\overrightarrow{x_{k}}, t\right)}{\rho}=\frac{\partial u_{k i}}{\partial t}+\frac{\partial\left(u_{k i} u_{k j}\right)}{\partial x_{k j}}+\frac{1}{\rho} \frac{\partial p_{k}}{\partial x_{k i}}-v \frac{\partial^{2} u_{k i}}{\partial x_{k j} \partial x_{k j}} .
$$

The Lagrangian force for the marker $x_{k}$ is determined using

$$
\frac{F_{i}\left(\overrightarrow{x_{k}}, t\right)}{\rho}=\frac{u_{k i}^{t+\Delta t}-u_{k i}+u_{k i}-u_{k i}^{t}}{\Delta t}+\left[\frac{\partial\left(u_{k i} u_{k j}\right)}{\partial x_{k j}}+\frac{1}{\rho} \frac{\partial p_{k}}{\partial x_{k i}}-v \frac{\partial^{2} u_{k i}}{\partial x_{k j} \partial x_{k j}}\right]^{t},
$$

where $u^{\sim}$ is a temporary parameter (Wang et al. [6]) and $\Delta t$ is the time step. Eqn (9) can be decomposed into two complementary equations

$$
\begin{gathered}
\frac{u_{k i}-u_{k i}^{t}}{\Delta t}+\left[\frac{\partial\left(u_{k i} u_{k j}\right)}{\partial x_{k j}}+\frac{1}{\rho} \frac{\partial p_{k}}{\partial x_{k i}}-v \frac{\partial^{2} u_{k i}}{\partial x_{k j} \partial x_{k j}}\right]^{t}=0, \\
\frac{F_{i}\left(\overrightarrow{x_{k}}, t\right)}{\rho}=\frac{u_{k i}^{t+\Delta t}-u_{k i}}{\Delta t}=\frac{\mathrm{u}_{\mathrm{i} \mathrm{IBM}}-u_{k i} i}{\Delta t},
\end{gathered}
$$

where $\mathrm{u}_{\mathrm{i} \text { IBM }}$ is the immersed boundary velocity obtained from the fluid-structure interaction. Under the effect of the force, the velocity on the Lagrangian marker $x_{k}$ at time $t+\Delta t\left(u_{k i}^{t+\Delta t}\right)$ can be modified to the desired velocity $\mathrm{u}_{\mathrm{i} \text { IBM }}$ (Wang et al. [6]).

A distribution function $D_{h}$ is applied to spread the two-way coupling between the Eulerian grid and the Lagrangian markers at the immersed boundary $x_{k}$. This distribution function is determined from its surrounding Eulerian grid nodes $x_{i}$. The velocity on the Lagrangian marker is calculated as

$$
\begin{gathered}
u_{k i}^{\tilde{k}}=\sum u_{i}^{*} D_{h}\left(\overrightarrow{x_{k \imath}}-\overrightarrow{x_{l}}\right) h^{2}, \\
D_{h}\left(\vec{x}_{k}-\vec{x}\right)=\frac{1}{h^{2}}\left[w_{g}\left(r_{x}\right) w_{g}\left(r_{y}\right)\right], \\
w_{g}(r)=\left\{\begin{array}{l}
\frac{3-2|r|+\sqrt{1+4|r|-4|r|^{2}}}{8} \text { for } 0<|r| \leq 1, \\
\frac{5-2|r|-\sqrt{-7+12|r|-4|r|^{2}}}{8} \text { for } 1<|r| \leq 2, \\
0 \text { for }|r|>2,
\end{array}\right.
\end{gathered}
$$


where $D_{h}$ is the distribution function, $h$ is the characteristic length associated to the Eulerian mesh size, $r_{x}=\frac{x_{k}-x}{h}$ and $r_{y}=\frac{y_{k}-y}{h}$.

The effect of the force on the Lagrangian markers which spreads into the Eulerian grid is expressed as

$$
f_{i}(\vec{x}, t)=\sum F_{i}\left(\overrightarrow{x_{k}}, t\right) D_{h}\left(\overrightarrow{x_{l}}-\overrightarrow{x_{k l}}\right) h^{\prime 2},
$$

where $h^{\prime}$ is the characteristic length associated to the Lagrangian mesh size.

The estimated velocity field from the first step of the two-step fractional method (eqn (4)), is updated to take into account the body presence. It can be written as

$$
u_{i}^{*}=u_{i}^{*}+\Delta t \frac{f_{i}}{\rho}
$$

During the process of interpolation to obtain the simulated velocity on the Lagrangian markers and extrapolation to spread the effect of the force on its surrounding Eulerian grids, the velocities on the Lagrangian markers may not be satisfied entirely. Therefore, the multidirect forcing technique, proposed by Wang et al. [6], is applied. This iterative technique is employed until the difference between the velocity simulated on the Lagrangian markers and the desired velocity approaches zero. The norm $L_{2}$ is used as the criterion for the loop process at the time $t+\Delta t$. The total Lagrangian force $F_{i \text { lag }}^{t+\Delta t}$, is the sum of all Lagrangian markers forces in all loops required to achieve the norm $L_{2}$ criterion at the time $t+\Delta t$.

\subsection{Structural model of the spring tethered system}

With reference to Fig. 2, assuming that the springs are perfectly elastic having negligible mass and drag properties and neglecting the cylinder's inertia, applying Newton's second law and Hooke's law, the following equations, for the resultant forces in each direction, result

$$
\begin{aligned}
& \sum F_{X \text { tot }}^{t+\Delta t}=F_{m x}^{t}-F_{x \text { lag }}^{t+\Delta t}, \\
& \sum F_{Y \text { tot }}^{t+\Delta t}=F_{m y}^{t}-F_{y \text { lag }}^{t+\Delta t},
\end{aligned}
$$

where

$$
\begin{aligned}
& F_{m x}^{t}=-F_{1}^{t} \cos \left(\beta_{1}{ }^{t}\right)+F_{2}^{t} \cos \left(\beta_{2}{ }^{t}\right)-F_{3}^{t} \sin \left(\beta_{3}{ }^{t}\right), \\
& F_{m y}^{t}=-F_{1}^{t} \sin \left(\beta_{1}{ }^{t}\right)-F_{2}^{t} \sin \left(\beta_{2}{ }^{t}\right)+F_{3}^{t} \cos \left(\beta_{3}{ }^{t}\right),
\end{aligned}
$$

where $F_{i l a g}$ is the total Lagrangian force acting on the cylinder at the time $t+\Delta t$ and $F_{m i}$ represents the resultant springs force at the time $t . \mathrm{F}_{1}, \mathrm{~F}_{2}$ and $\mathrm{F}_{3}$ are calculated by the Hooke's law at the time $t$. The cylinder center position at the time $t$ is given by $(x, y)=\left(x^{t}, y^{t}\right)$ and the geometrical domain center is given by $(\mathrm{x}, \mathrm{y})=\left(\mathrm{x}_{\mathrm{cen}}, \mathrm{y}_{\mathrm{cen}}\right)$, thus, the angles values and the springs forces are calculated as follows

$$
\begin{gathered}
\beta_{1}^{\mathrm{t}}=\tan ^{-1}\left(\frac{\mathrm{y}^{\mathrm{t}}}{\mathrm{x}^{\mathrm{t}}}\right), \\
\beta_{2}^{\mathrm{t}}=\tan ^{-1}\left(\frac{\mathrm{y}^{\mathrm{t}}}{2 \mathrm{x}_{\operatorname{cen}-\mathrm{x}^{\mathrm{t}}}}\right), \\
\beta_{3}^{\mathrm{t}}=\tan ^{-1}\left(\frac{\mathrm{x}^{\mathrm{t}}-\mathrm{x}_{\operatorname{cen}}}{2 \mathrm{y}_{\operatorname{cen}}-\mathrm{y}^{\mathrm{t}}}\right), \\
F_{1}^{t}=k l_{1}^{t},
\end{gathered}
$$




$$
\begin{aligned}
& F_{2}^{t}=k l_{2}^{t}, \\
& F_{3}^{t}=k l_{3}^{t},
\end{aligned}
$$

where the displacement by the springs, $l_{\mathrm{i}}^{\mathrm{t}}$, is calculated by the cylinder center position at the time $t$ and the springs equilibrium position which coincides with the geometrical domain center (illustrated in Section 4), calculated as follows

$$
\begin{gathered}
l_{1}^{t}=\sqrt{\left(\mathrm{x}^{\mathrm{t}}\right)^{2}+\left(\mathrm{y}^{\mathrm{t}}\right)^{2}}-\sqrt{\left(\mathrm{x}_{\text {cen }}\right)^{2}+\left(\mathrm{y}_{\text {cen }}\right)^{2}}, \\
l_{2}^{t}=\sqrt{\left(2 \mathrm{x}_{\text {cen }}-\mathrm{x}^{\mathrm{t}}\right)^{2}+\left(\mathrm{y}^{\mathrm{t}}\right)^{2}}-\sqrt{\left(\mathrm{x}_{\text {cen }}\right)^{2}+\left(\mathrm{y}_{\text {cen }}\right)^{2}}, \\
l_{3}^{t}=\sqrt{\left(\mathrm{x}^{\mathrm{t}}-\mathrm{x}_{\text {cen }}\right)^{2}+\left(2 \mathrm{y}_{\text {cen }}-\mathrm{y}^{\mathrm{t}}\right)^{2}}-\sqrt{\left(\mathrm{y}_{\text {cen }}\right)^{2}} .
\end{gathered}
$$

After calculating the total force acting on the cylinder, it is possible to determine the components of acceleration

$$
\begin{aligned}
\ddot{x}^{t+\Delta t} & =\frac{\sum F_{X t o t}^{t+\Delta t}}{m}, \\
\ddot{y}^{t+\Delta t} & =\frac{\sum F_{Y \text { tot }}^{t+\Delta t}}{m} .
\end{aligned}
$$

These accelerations are integrated to estimate the velocities of the body

$$
\begin{aligned}
& \mathrm{u}_{\text {IBM }}=\dot{x}^{t+\Delta t}=\dot{x}^{t}+\ddot{x}^{t+\Delta t} \Delta t, \\
& \mathrm{v}_{\text {IBM }}=\dot{y}^{t+\Delta t}=\dot{y}^{t}+\ddot{y}^{t+\Delta t} \Delta t .
\end{aligned}
$$

Integrated again to determine the new cylinder center

$$
\begin{aligned}
& x^{t+\Delta t}=x^{t}+\dot{x}^{t+\Delta t} \Delta t, \\
& y^{t+\Delta t}=y^{t}+\dot{y}^{t+\Delta t} \Delta t .
\end{aligned}
$$

The computational algorithm is described in the flow chart on the following pages.

\section{PROBLEM DESCRIPTION}

The problem consists of a square two-dimensional cavity of width $l_{x}$ and height $l_{y}$, with $l_{x}=$ $l_{y}=L=1 \mathrm{~m}$, where the upper wall moves from left to right with a given velocity $\mathrm{U}=$ $1.0 \mathrm{~m} / \mathrm{s}$ which drives the flow and $U=V=0 \mathrm{~m} / \mathrm{s}$ on the remaining three sides. The cylinder with diameter $D=0.1 \mathrm{~m}$ is sustained by a set of three springs, as depicted in Fig. 2. Initially the fluid is at rest and the cylinder is in the equilibrium position at the center of the domain. The Reynolds number is defined based on the characteristic length, $L=1 \mathrm{~m}$ and the characteristic velocity $U=1.0 \mathrm{~m} / \mathrm{s}$. The density of the fluid, $\rho=1 \mathrm{~kg} / \mathrm{m}^{3}$ and the kinematic viscosity is the variable responsible to change the Reynolds number $(v=U L / R e)$.

\section{RESULTS}

The numerical platform was validated by the simulations of the classical lid-driven cavity flow without the presence of the immersed body. The spring system was then validated. The final step involved the fluid-structure interaction simulation. 


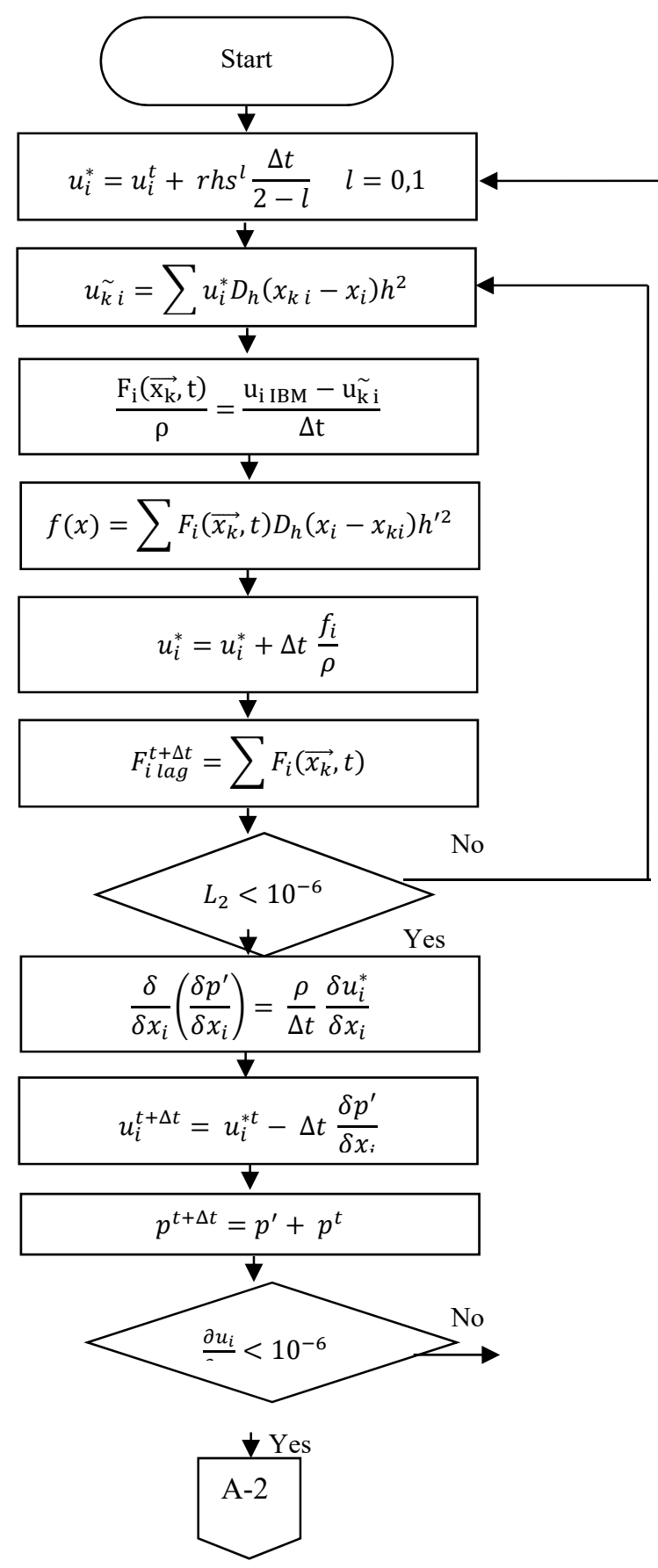



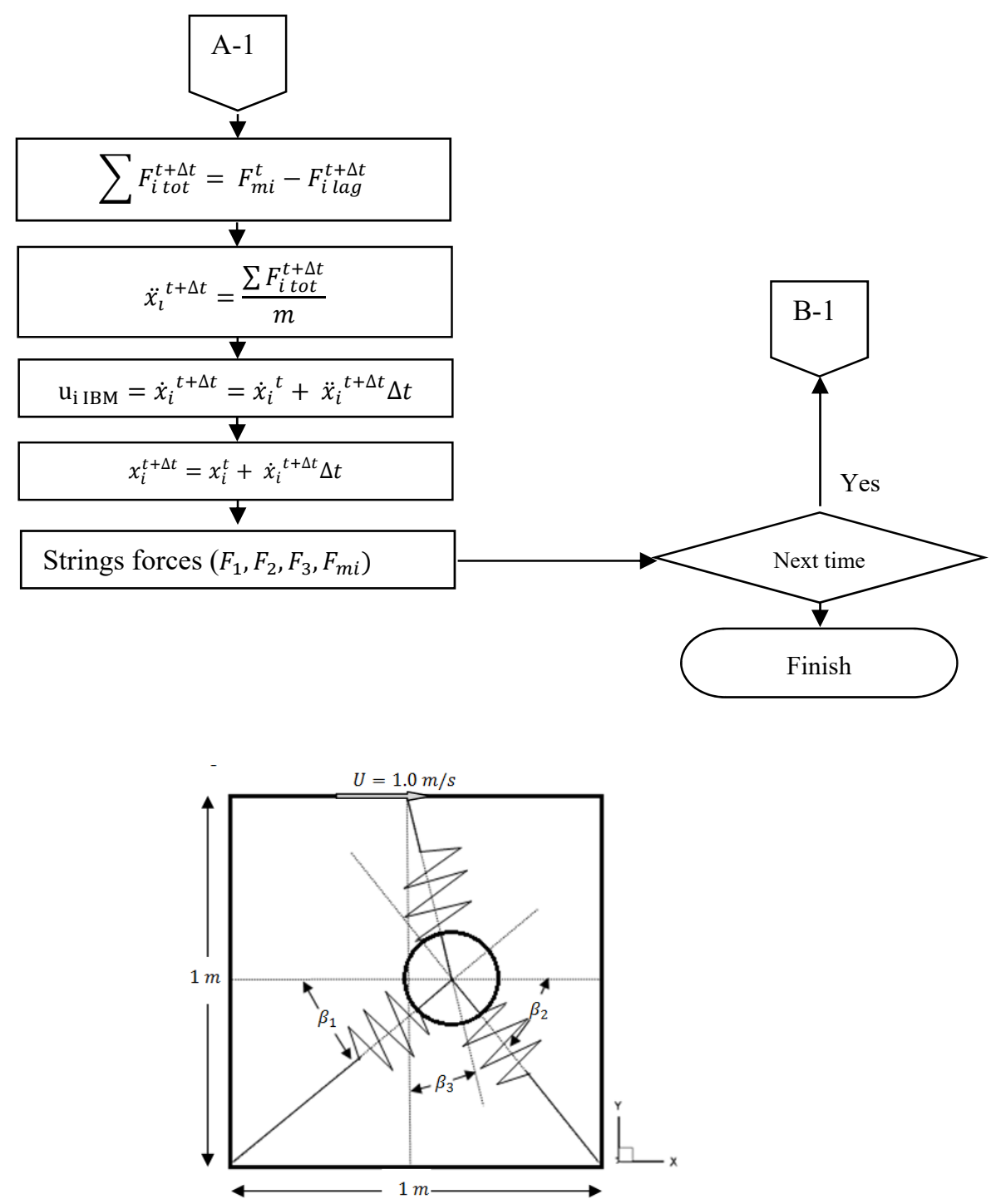

Figure 2: Problem proposal scheme with the cylinder in an unbalanced position.

For the lid-driven cavity flow without the presence of the immersed body, the simulations were performed for Reynolds numbers of 100, 400 and 1,000. Uniform rectilinear meshes of $129 \times 129,141 \times 141$ and $160 \times 160$ were used. Ghia et al. [17] reported the first benchmark results for the classical problem of the two-dimensional, incompressible lid-driven cavity flow. The results include the flow patterns, structures identification, minimum and maximums of several fields and velocity profiles passing through the geometric center of the cavity. This data was used to compare and validate the present numerical model.

The characteristics and topology of the Lid-driven cavity flow depend on the Reynolds number. For $R e=100$ the flow structure is characterized by a primary eddy centred slightly 
to the upper right side of the cavity. In addition to this eddy, there are two smaller eddies at the bottom left and at the bottom right corners of the cavity. As the Reynolds number increases the secondary eddies grow in size and the primary eddy adapts to this change, shifting towards the center of the cavity. Fig. 3 shows the streamlines pattern for primary and secondary eddies associated with the velocity vector magnitude $\left(\sqrt{u^{2}+v^{2}}\right)$. As the Reynolds number increases the flow becomes more energized and the primary eddy is circumvented by this region.

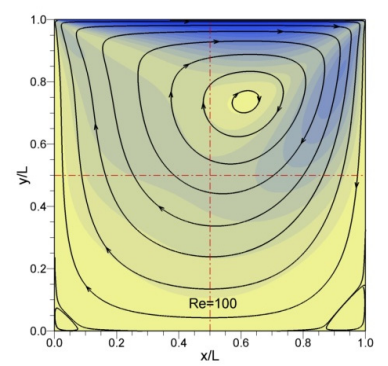

(a)

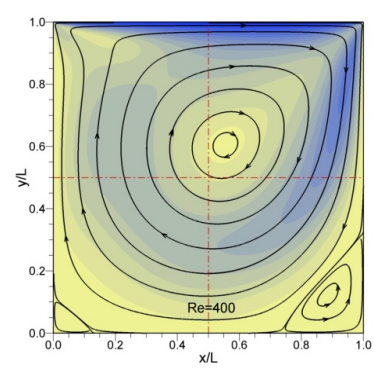

(b)

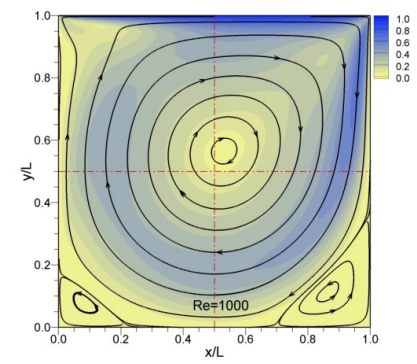

(c)

Figure 3: Streamlines pattern for primary and secondary eddies associated with the velocity vector magnitude for: (a) $\operatorname{Re}=100$; (b) $\operatorname{Re}=400$; and (c) $\operatorname{Re}=1000$.

In order to validate the spring system equations, the cylinder was initially placed in an arbitrary position, such as $x / L=0.4$ and $y / L=0.6$. The cylinder's center position was then tracked against dimensionless time $(t *=t /(L / U))$ as shown in Fig. 4. Since no damping is present, the cylinder continues to move indefinitely.

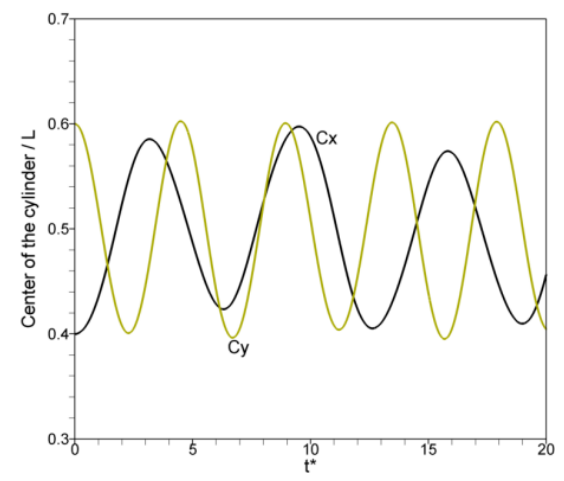

(a)

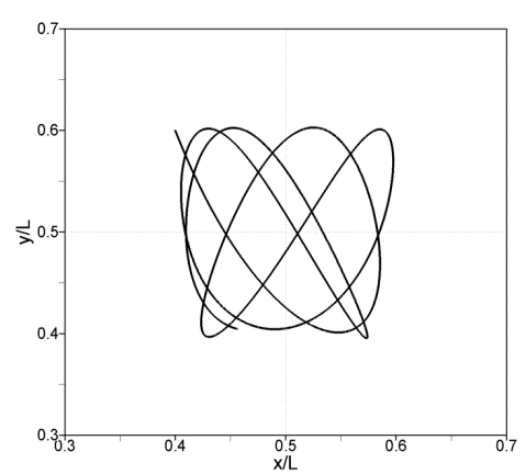

(b)

Figure 4: History of the cylinder center position at $\mathrm{k}=0.1 \mathrm{~N} / \mathrm{m}$. (a) With respect to time; and (b) With respect to space.

For the fluid-structure interaction, the square cavity was discretized using a mesh size of $160 \times 160$ volumes. The simulations were run at Reynolds numbers of $\operatorname{Re}=100,400$ and 
1,000 and spring constants $k=0.01,0.1$ and $1.0 \mathrm{~N} / \mathrm{m}$. The cylinder diameter was fixed at D $=0.1 \mathrm{~m}$. With the fluid initially at rest and the cylinder in the equilibrium position $(x / L=$ $y / L=0.5)$, the simulations were run for $100 \mathrm{~s}$.

Results show that the transient cylinder center position depend on the values of $\mathrm{k}$ and Re. As shown in Fig. 5, there are two distinct results related to the $k$ value. When the spring constant is small, $k=0.01 \mathrm{~N} / \mathrm{m}$, the resultant springs force is much weaker than the fluid dynamics forces (Fig. 5(a)) and the springs stretch freely resulting in the cylinder motion mainly affected by the fluid dynamics action. On the other hand, when the spring constant is larger, a clear response from the springs forces interacting with the fluid dynamics is evident.

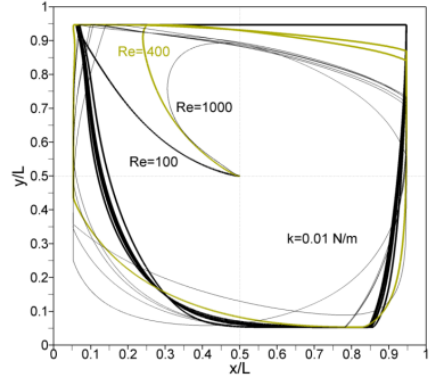

(a)

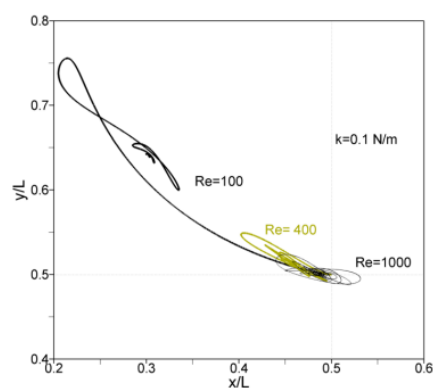

(b)

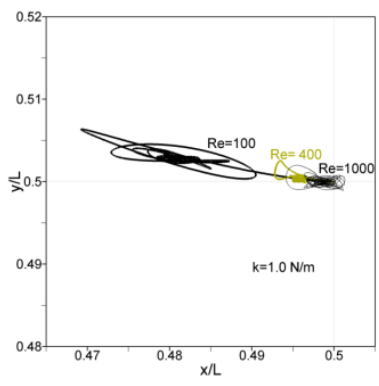

(c)

Figure 5: The cylinder center position along the time for spring constant. (a) $k=0.01 \mathrm{~N} / \mathrm{m}$; (b) $\mathrm{k}=0.1 \mathrm{~N} / \mathrm{m}$; and (c) $\mathrm{k}=1 \mathrm{~N} / \mathrm{m}$.

\section{CONCLUSIONS}

In this paper, a simplified fluid-structure interaction model consisting of a cylinder tethered by a spring system interacting dynamically with the incompressible two-dimensional liddriven cavity flow using the immersed boundary (IB) method was investigated. A structured Cartesian grid was employed for the Eulerian domain while the boundaries were represented through Lagrangian markers. The advantage of this method is that bodies of almost any arbitrary shape can be added without grid restructuring, a procedure which is often timeconsuming. The numerical implementation of a Cartesian grid is simpler to implement than an alternative body-fitted coordinates grid.

Using the IB method, the motion of the cylinder does not impose grid restructuring at each time step when FSI is present. Additionally the partitioned approach used in this study shows an efficient way to couple the flow and structural solver.

From the cases studied, cases having large spring constants, strings forces induce the cylinder to an oscillatory motion damped by the fluid viscous forces, consequently the amplitude of the displacements decrease.

It can therefore be concluded that the immersed boundary method is a good tool to model fluid-structure interaction problems. Additionally this method can also accommodate complex geometry without the need of complex grid creation algorithms.

\section{ACKNOWLEDGEMENTS}

The authors thank the College of Mechanical Engineering (FEMEC) of Federal University of Uberlandia (UFU), Federal University of Mato Grosso (UFMT), Federal University of Technology - Paraná (UFTPR) and University of Malta for the support. 


\section{REFERENCES}

[1] Ferziger, J.H. \& Peric, M., Computational Methods for Fluid Dynamics, 3rd ed., Springer-Verlag: Berlin and New York, 2002.

[2] Peskin, C., Flow patterns around heart valves: A numerical method. Journal of Computational Physics, 10, pp. 252-271, 1972.

[3] Lima e Silva, A.L.F., Silveira-Neto, A. \& Damasceno, J., Numerical simulation of two-dimensional flows over a circular cylinder using the immersed boundary method. Journal of Computational Physics, 198, pp. 351-370, 2003.

[4] Uhlmann, M., An immersed boundary method with direct forcing for the simulation of particulate flows. Journal of Computational Physics, 209, pp. 448-476, 2005.

[5] Vanella, M. \& Balaras E., A moving-least-squares reconstruction for embeddedboundary formulations. Journal of Computational Physics, 228(18), pp. 6617-6628, 2009.

[6] Wang, Z., Fan, J. \& Luo, K., Combined multi-direct forcing and immersed boundary method for simulating flows with moving particles. International Journal of Multiphase Flow, 34, pp. 283-302, 2007.

[7] Borges, J.E., Lourenço, M.A.S., Padilla, E.M. \& Micallef, C., Immersed boundary method application as a way to deal with the three-dimensional sudden contraction. Computation, 6(3), p. 50, 2018.

[8] Ha, S.T., Ngo, L.C., Saeed M., Jeon, B.J. \& Choi, H., A comparative study between partitioned and monolithic methods for the problems with 3D fluid-structure interaction of blood vessels. Journal of Mechanical Science and Technology, 31, pp. 281-287, 2017.

[9] Degroote, J., Haelterman, R., Annerel, P., Bruggeman, P. \& Vierendeels, J., Performance of partitioned procedures in fluid-structure interaction. Computers and Structures, 88, pp. 446-457, 2010.

[10] Degroote, J., Bathe, K. \& Vierendeels, J., Performance of a new partitioned procedure versus a monolithic procedure in fluid-structure interaction. Computers and Structures, 87(11-12), pp. 793-801, 2009.

[11] Chern, M.J., Kuan, Y.H., Nugroho, G., Lu, G.T. \& Horng, T.L., Direct-forcing immersed boundary modeling of vortex-induced vibration of circular cylinder. Journal of Wind Engineering and Industrial Aerodynamics, 134, pp. 109-121, 2014.

[12] Dettmer, W. \& Perić, D., A computational framework for fluid-rigid body interaction: Finite element formulation and applications. Computer Methods in Applied Mechanics and Engineering, 195(13-16), pp. 1633-1666, 2006.

[13] Williamson, C.H.K. \& Govardhan, R., Vortex-induced vibrations. Annual Review of Fluid Mechanics, 36, pp. 413-455, 2004.

[14] Campregher, R., Mathematical modeling for three-dimensional fluid-structure interaction problems. Doctoral thesis, University Federal of Uberlandia, 2005.

[15] Patankar, S., Numerical Heat Transfer and Fluid Flow, Series in Computational and Physical Processes in Mechanics and Thermal Sciences, 1st ed., Hemisphere Publishing Company, 1980.

[16] Kim, J. \& Moin, P., Application of a fractional step method to incompressible NavierStokes equations. Journal of Computational Physics, 59, pp. 308-323, 1985.

[17] Ghia, U., Ghia, K.N. \& Shin, C.T., High-re solutions for incompressible flow using the Navier-Stokes equations and a multigrid method. Journal of Computational Physics, 48, pp. 387-411, 1982. 